\title{
Path Estimation and Motion Detection of Moving Object in Videos
}

\author{
Ritika $^{1}$, Gianetan Singh Sekhon ${ }^{2}$ \\ ${ }^{1}$ M.Tech CE, Ycoe, Guru Kashi Campus/ Punjabi University, Talwandi Sabo \\ ${ }^{2}$ Assistant Professor, Ycoe, Guru Kashi Campus/ Punjabi University, Talwandi Sabo
}

\begin{abstract}
This paper discusses an efficient and effective approach for identifying and tracking of moving object from a video. A video is captured by stationary camera. Moving object tracking and detection from video sequences has applications in several areas such as automatic video surveillance, motion-based recognition, video indexing, human-computer interaction, traffic monitoring, and vehicle navigation. In this work, we present a computer vision-based approach for object tracking and detection. A method is proposed to detect and track moving object through video even if background is changed at any instant and capable of plotting a $3 D$ graph mesh based on the moving object in between any number of frames per second. We use consecutive frame analysis technique to detect background changing criteria and use morphological filtering for image enhancement. Finally, we will get the co-ordinates for the moving object and these co-ordinates are imported to any other 3D software's like MAYA etc to analyze or edit the results calculated by the algorithm.
\end{abstract}

Keywords- Object tracking, Object detection, Motion estimation, Computer vision.

\section{Introduction}

Tracking targets in video has been one of the most important problems in computer vision and has attracted more and more researchers working on it [1]. In general, there are three approaches to analyze moving object: one is object tracking aim at extracting characteristics such as point tracking, kernel tracking, silhouette tracking, Second is object representation aim at extracting characteristics such as points, primitives geometric shapes, object silhouette and contour, articulated shape models, skeletal models and third is object detection aim at extracting characteristics such as point detectors, segmentation, background subtraction.

The automatic detection of moving objects in monitoring system needs efficient algorithms. The common method is simple background subtraction i.e. to subtract current image from background. But it can't detect the difference when brightness difference between moving objects and background is small. The other approach is to use some algorithms such as color based subtraction technique but the costs are very high and have problem in stability.

Here a method is proposed to detect moving objects using consecutive frames evaluation technique. The objective is to provide software that is able to track and detect the moving object in any video even if background is change at any instant and performs tracking along a mesh graph in 3D which can be further used in any pc. The algorithm is implemented in MATLAB and the results demonstrate that both the accuracy and processing speed are very promising.

\section{Related Work}

There is much research work in the field of object tracking in videos over the past decades. Some of the work done has been discussed as presented an approach for handling target detection and tracking in dynamic scenes, in which, motion compensation is generated by pyramidal optical flow [1], a new contour based tracking method using active contour [2], an adaptive tracking algorithm for lung tumors in fluoroscopy using online learned collaborative trackers. No shape or motion priors are required for this tracking algorithm [3], shape based object recognition in Videos Using 3D Synthetic Object Models [4], a method of moving object detection on moving platforms is proposed. This method composed of moving blob detection and shape refinement phases to provide robust moving object detection result [5], propose a method to identify and track an object of interest within compressed MPEG-2 video using only motion information [6], estimating 3D geometry and motion of dynamic scenes based on captured stereo sequences. A dynamic programming based technique is used [7], presents perceptual organization based method for real-time license plate identification and tracking [8], Presents a novel motion estimation method for mesh-based video motion tracking. The method called meshbased square-matching (MB-SM) motion estimation method [9], effective approach to simultaneously identifying and tracking two free moving and gesturing hands, together with the provision of accurate segmentation masks of the hands regions [10], presents an object tracking method in diving video sequences by particle filter with multiple motion models. In this method, video paragraphing based on Hough transform technique [11], present a computer vision-based approach for event detection and data collection at traffic 
intersections [12], proposed a algorithm of a new high speed execution technique of the level set method named the Fast Level Set Method (FLSM) [13].

\section{The Proposed Algorithm}

Detection of moving objects in video is one of the most important and fundamental technologies to develop the real world computer vision systems, such as video monitoring system, intelligent-highway system, intrusion surveillance, etc. The usual method for detecting moving objects is simple background subtraction that is to subtract current image from background image. Simple background subtraction is susceptible to sudden changes. And when the brightness difference between moving objects and the background is small, it cannot detect the difference. In order to resolve these problems, some algorithms such as color based subtraction technique and the technique based on optical flows have been proposed. But the computational costs of these methods are very high and have problem in stability [18].

We use consecutive frame evaluation technique for moving objects detection. This approach uses the motion to distinguish moving objects from the background. So it is more efficient than the previous approaches. Consecutive frame evaluation approach is robust to environmental changes, however, unable to detect motionless objects.

Fig. 1 shows the overall scheme of the proposed algorithm. In our system, video is captured with a stationary camera. Our algorithm does not want any manual input for starting object tracking. We plot object tracking in real time and logging the data into memory frame via frame. According to our algorithm first frame remains constant at the end of the video as it is used to calculate the per pixel distance (in $\mathrm{mm}$ ). We calculate the changing background with moving object and compare each frame with the previous one. But the computational costs of these methods are very high and have problem in stability [18].

The state of single object is estimate by morphology filter. Morphology is formulated in terms of set theory. Sets represent objects in an image; for instance, the set of all white pixels in a binary image is a complete morphological description of an image. We use morphological filtering for image enhancement. The goals of image enhancement include the improvement of the visibility and perceptibility of the various regions into which an image can be partitioned and of the detectability of the image features inside these regions. These goals include tasks such as: cleaning the image from various types of noise; enhancing the contrast among adjacent regions or features; simplifying the image via selective smoothing or elimination of features at certain scales and retaining only features at certain desirable scales [19].

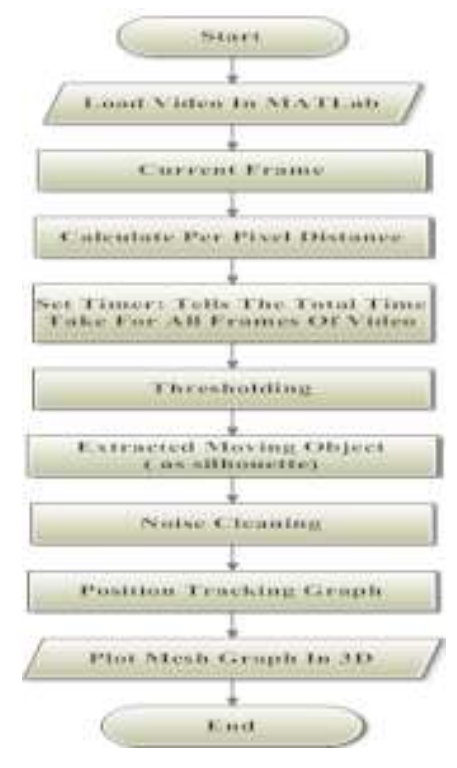

Fig. 1 the proposed algoritm

The algorithm is based on calculating per pixel distance (in $\mathrm{mm}$ ). We use a scale to find the dimensions of the moving object. We have a real time video and in this video moving object is a car. The dimensions of the car are calculated by the scale (in mm). After then distance in pixels is calculated with distance formula. The distance formula is given below:

$$
d=\sqrt{\left(x_{2}-x_{1}\right)^{2}+\left(y_{2}-y_{1}\right)^{2}}
$$


The scale tells the dimensions of the car with co-ordinates $\left(x_{1}, y_{1}\right)\left(x_{2}, y_{2}\right)$. We use the scale is of $300 \mathrm{~mm}$, which is the actual distance. Now per pixel distance is calculated in $\mathrm{mm}$ by distance in pixels / actual distance.

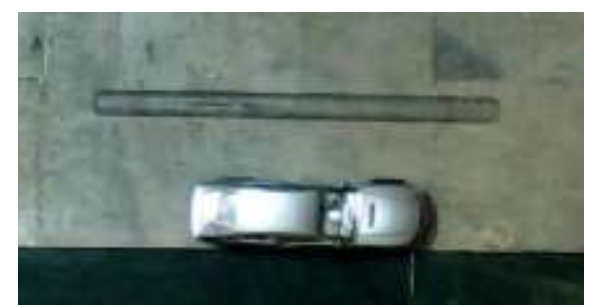

Fig. 2 distance calculation

To calculate the total time taken by each frame of the video, we set a timer. It tells the total time taken during execution of all frames. The calculated time will vary according to the configuration of the user's system. To set a threshold value, we select the maximum value of the pixel out of all the pixels. Then the moving object is extracted from the video. A position tracking graph is plotted which is used to plot the mesh graph in 3D as a result.

\section{Assumptions}

\section{Experimental Results}

1. The camera that is capturing videos is fixed.

2. Single object tracking is there.

3. The movement of object is smooth and not abrupt.

Fig. 3 shows the extraction of moving object from the video after image enhancement by using morphological filtering. Fig. 4 shows the position tracking graph using proposed algorithm. Fig. 5 shows the total time taken for execution of all frames, the total time can vary according to the system configuration and Fig. 6 shows the mesh graph in 3D as $\mathrm{x}$-axis shows the distance covered in $\mathrm{mm}, \mathrm{y}$-axis shows the distance covered in $\mathrm{mm}$ and $\mathrm{z}$-axis shows the number of frames.



Fig. 3 extracted moving object from the video

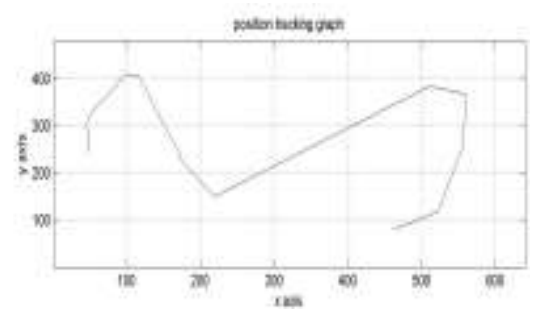

Fig. 4 position tracking graph

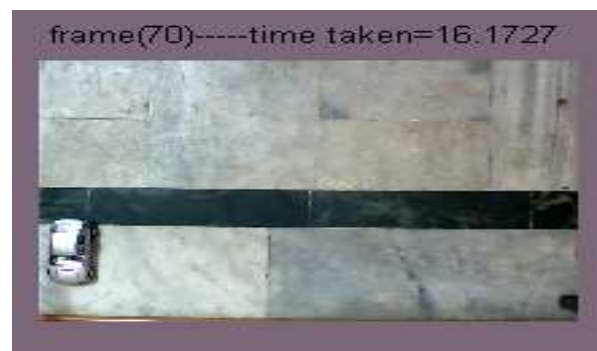

Fig. 5 total time taken by all frames www.iosrjournals.org 


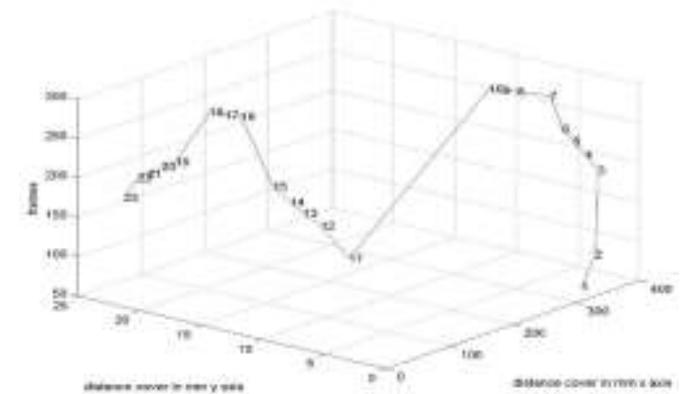

Fig. 6 mesh graph in 3D

\section{Conclusion}

We have proposed tracking of moving object from a video algorithm using consecutive frame evaluation technique. Video is captured with a stationary camera. An important component in such systems is the ability for a computer to track and identify moving objects. Till now in many proposed works, there is plotting the movement of objects through videos but none of them is capable of plotting graph of the moving object if the background is changed at any instant, hence our algorithm is capable to plot the movements of object through videos if the background is changed at any instant.

The problem of tracking the movement of a desired object is captured by a real time video. We have improved the accuracy and provided efficient way to track moving object from a real time video and also plotted the mesh graph in 3D of the extracted moving object from the video.

The co-ordinates imports to any other 3D software like MAYA or RIHNOSOUR to analysis or edit with the results calculated by the algorithm. The objective is to provide software that can be used on a PC for performing object tracking along with video enhancement. The algorithm improves the detection and tracking of moving objects in the real time video. It will be very helpful in the video based applications such as automatic video surveillance, animations etc where the moving/running objects/vehicles can be easily tracked.

\section{References}

[1] Pengwei LIU, Huiyuan WANG et al. "Motion Compensation Based Detecting and Tracking Targets in Dynamic Scene”, IEEE, pp.703-706, 2010.

[2] Sajjad Torkan, Alireza Behrad “A New Contour Based Tracking Algorithm Using Improved Greedy Snake”, IEEE, 2010

[3] Baiyang Liu, Lin Yang et al. "An Adaptive Tracking Algorithm Of Lung Tumors In Fluoroscopy Using Online Learned Collaborative Trackers”, IEEE, pp.209-212, 2010.

[4] Alexander Toshev, Ameesh Makadia, Kostas Daniilidis "Shape-based Object Recognition in Videos Using 3D Synthetic Object Models", IEEE, pp.288-295, 2009.

[5] Ming-Yu Shih, Yao-Jen Chang, Bwo-Chau Fu, and Ching-Chun Huang “Motion-based Background Modeling for Moving Object Detection on Moving Platforms", IEEE, pp.1178-1182, 2007.

[6] Mark Ritch, Nishan Canagarajah “Motion-Based Video Object Tracking In The Compressed Domain”, IEEE, pp-301-304, 2007.

[7] Minglun Gong “A GPU-based Algorithm for Estimating 3D Geometry and Motion in Near Real-time”, IEEE, 2006.

[8] Huiqiong Chen, Derek Rivait and Qigang Gao "Real-Time License Plate Identification by Perceptual Shape Grouping and Tracking”, IEEE, pp.1352-1357, 2006.

[9] Mohammed Sayed and Wael Badawy “A novel motion estimation method for mesh-based video motion tracking”, IEEE, pp.337$340,2004$.

[10] Li-Qun Xu "Simultaneous Tracking And Segmentation Of Two Free Moving Hands In A Video Conferencing Scenario”, IEEE, pp.49-52, 2003.

[11] Beiji Zou, Xiaoning Peng and Liqin Han "Particle Filter With Multiple Motion Models For Object Tracking In Diving Video Sequences", IEEE, pp.224-228, 2008.

[12] Harini Veeraraghavan, Paul Schrater and Nikolaos Papanikolopoulos "Switching Kalman Filter-Based Approach for Tracking and Event Detection at Traffic Intersections", IEEE, pp.1167-1172, 2005.

[13] Yumi Iwashita, Ryo Kurazume et al. “Fast Implementation of Level Set Method and Its Real time Applications”, IEEE, pp.63026307, 2004.

[14] Min-Cheng Pan, Yau-Liang Tsai and Ying-Jieh Huang "Motion Tracking of Multi-objects for Access Surveillance Based on the Panoramic Imaging System”, IEEE, pp.208-215, 2003.

[15] Yankun Wei and Wael Badawy "On Reducing The Inter Frame rate: An Embedded Residual Image Model For Mesh-Based Motion Tracking”, IEEE, pp.677-680, 2002.

[16] Hao Jiang and Mark S. Drew “A Predictive Contour Inertia Snake Model For General Video Tracking”, IEEE, pp.413-416, 2002.

[17] Jiingecin Lim, Hong K. Cho, and Jong Beom Rat “An Improved Video Object Tracking Algorithm Based On Motion Reestimation”, IEEE, pp.339-342, 2000.

[18] Rajan Sehgal "Video Image Enhancement and Object Tracking”, A Thesis, 2006.

[19] Petros Maragos “Morphological Filtering For Image Enhancement And Feature Detection”, 2004. 